\title{
IX. \\ Sur la conception aristotélicienne de la causalité.
}

Ton

Leon Robin - Caen.

II.

$\S 27$. Cette identité de la cause avec la quiddité ou la forme sera rendue plus évidente encore, si on se rappelle ce qui a été dit précédemment à propos du moyen-terme ( $\$ 4-7)$, que dans les cas même où nous avons affaire à la cause matérielle, ou bien à la cause motrice, ou enfin à la cause finale, c'est toujours en définitive la forme ou l'essence qui est la cause véritable du fait. Le rapport de chacune de ces sortes de cause à son effet peut être remplacé par le rapport de la forme, ou de la quiddité, à ce dont elles sont la forme ou la quiddité et qu'elles servent à définir. Or ce second terme, qu'est-il par rapport à la notion totale? Il en est la partie matérielle, ce qui n'est que puissance sans la causalité de la forme. La lune a des éclipses, c'est un fait; mais le fait de l'éclipse ne représente pour moi, par rapport à la lune, rien de plus qu'une possibilité tout à fait indéterminée, tant que j'ignore l'essence ou la quiddité du phénomène. Quand je saurai qu'elle consiste en une interposition de la terre, j'aurai découvert la cause et déterminé du même coup quelle forme comporte cette matière par rapport au sujet et quel est l'acte dont elle est la puissance. Le sujet lui-même, bien qu'il soit, à vrai dire, une substance composée de matière et de forme, n'en est pas moins, lui aussi, du point de vue où nous l'envisageons et en tant que capable de telles ou telles déterminations non encore reconnues, un aspect ou un élément de la .puissance. En résumé nous 
pouvons remplacer le rapport de la cause à l'effet par celui de la forme à la matière, ou de l'acte à la puissance; de même que ces deux termes sont liés indissolublement dans la constitution des substances, de même il y a union nécessaire de la cause et de l'effet dans la production de tel phénomène ou dans la présence de telle propriété èn un sujet déterminé, et l'effet est une puissance actualisée par. le cause, comme la matière l'est par la forme. Chercher le pourquoi d'un fait complexe ou d'une chose concrète, c'est chercher quelle est la quiddité ou la forme de telle ou telle matière. .

Cette doctrine est exposée principalement dans deux passages, l'un des Seconds Analy tique s II, 8, l'autre de la Mé ta physique Z, 17. - Dans le premier (93 a, 9-13), Aristote explique qu'il y a une façon de démontrer l'essence sans cercle vicieux,

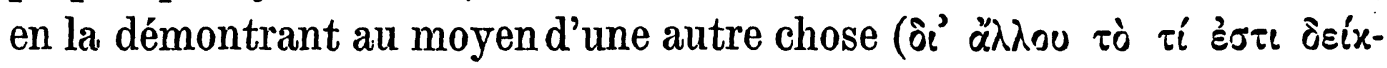

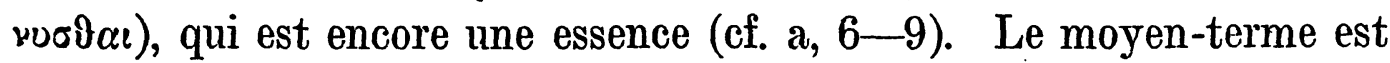
done nécessairement essence (et, par conséquent, universel) s'il s'agit de démontrer l'essence; il est un propre s'il s'agit de démontrer le propre.

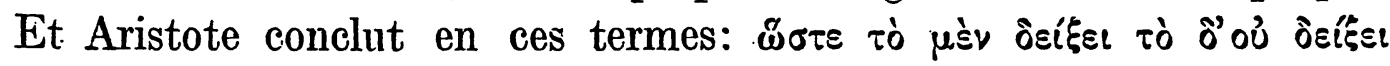

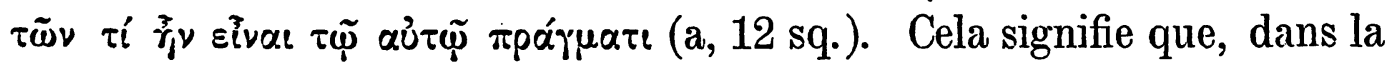
quiddité totale d'une même chose, il y a une partie que l'on ne pourra pas démontrer, c'est celle qui sert de moyen à la démonstration - et une partie que l'on pourra démontrer, c'est celle qui se conclut de la première. Or le ri îjv sivva ou la quiddité, ce n'est pas, au fond et à considérer l'essence en elle-même, la forme toute seule et prise abstraitement à part de la matière. C'est la forme en tant qu'elle détermine sa matière et qu'elle constitue la réalité propre de chaque essence totale, sa réalité complète et première, forme et matière, différences et genre. Par conséquent le $\tau i$ j̉v eival, c'est le défini: il est ce qu'il y a de propre dans l'essence du défini, cet aspect de l'essence sous lequel elle est pensée sans aucune possibilité d'erreur ${ }^{38}$ ).

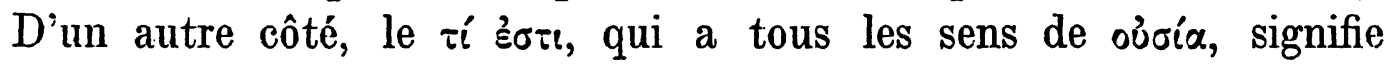
comme ce dernier terme non pas seulement la substance en tant

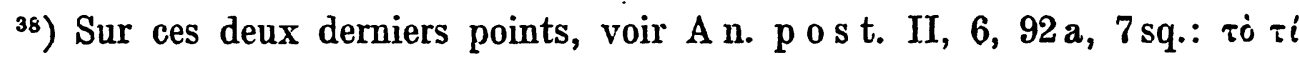

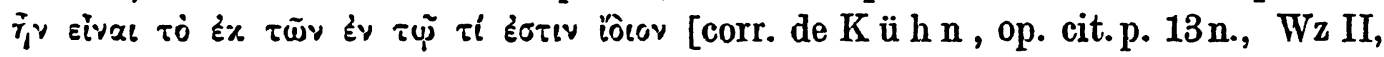
390, au lieu de ìíwv] (cf. T o p. I, 4, 101 b, 19 sq.) et D e A n. III, 6, 430 b, 28:

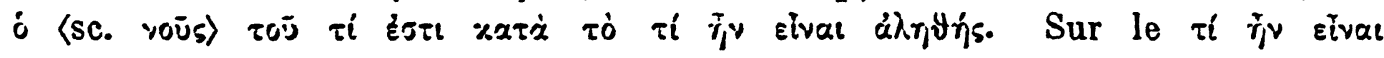
cf. Wz 0 r g. II, 399-401, . Bz In d. 764 a, 50 sqq. et aussi la n. 24 de mon livre déjà cité, p. 28 sq. 
qu'existante, mais aussila définition tout entière, et la forme seule, et même . la matière seule. Il se confond done avec le $\tau^{\prime} \tilde{j}_{j} v$ eiva dans la seconde et la troisième signification, mais il n'en est pas de même ni pour la première, ni pour la dernière, qui nous intéresse tout particulièrement.

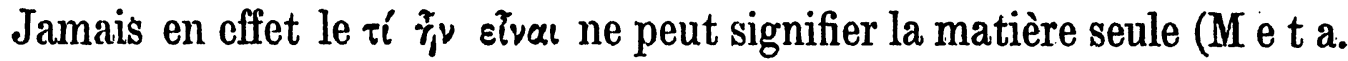
$\mathrm{Z}, 7,1032 \mathrm{~b}, 14)$; le $\tau i ́$ É $\sigma \tau$ le peut au contraire (B z, In d. $763 \mathrm{~b}$, 21 sq.); si d'ailleurs il désigne principalement le genre (i b i d., b, 39 sqq.), il représente par là même l'élément matériel de la définition (M e t a. $\Delta, 6,1016$ a, 26; 24, 1023 b, 2; 28, 1024 b, 8 sq. ; Z, 7, 1033 a, 3 sq.; 12, 1038 a, 6 sq. ; H, 3, 1043 b, 10-14; I, 8, 1058 a, 23 sq.) et, pour la même raison, il peut avoir plus d'extension que le défini. Mais

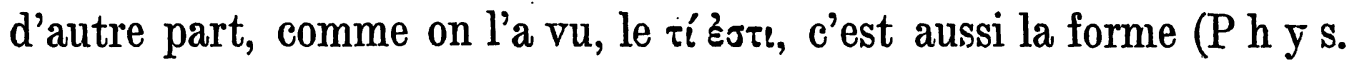
II, 2, 194 b, 10; 7, 198 a, 25, b, 3; M e t a. A, 6, 988 a, 10 et al.) et

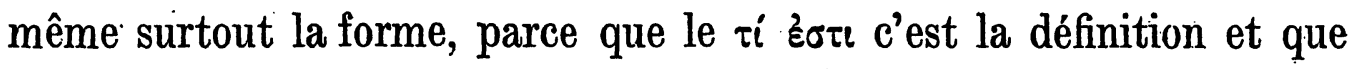
la forme est le principal dans toute définition. De tout ce qu'on vient de dire, il résulte donc que dans l'unité synthétique du $\tau^{\prime} \eta^{2} v$ eivar on peut distinguer une essence formelle et une essence matérielle, ou, sil'on veut, une partie formelle et une partie matérielle del'essence.

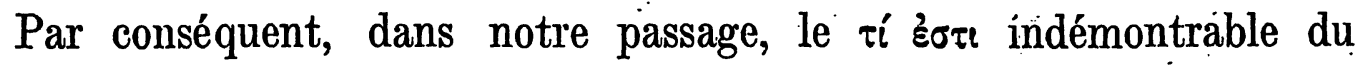
$\tau i$ j̃v عiva de départ (A n. p o s t. I, 2, 72 a, 7 sq.; 3, 72 b, 18-22; II, 3, 90 b,

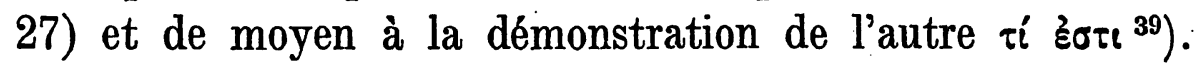

Le second texte (M e ta. Z, 17, 1041 b, 4-28) n'est pas moins significatif. „Puisqu'il faut, dit Aristote, que le fait soit donné d'abord et qu'il existe [avant de chercher la cause] ${ }^{40}$ ), il est clair que la

39) C'est ce que Phil op on exprime en excellents termes: ... عís $\mu \dot{\varepsilon} \nu$ ìj

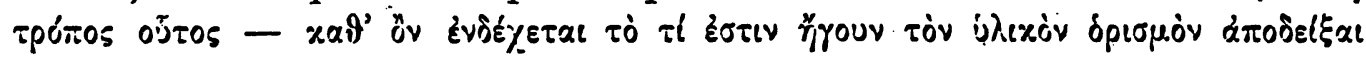

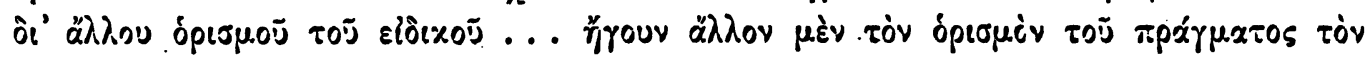

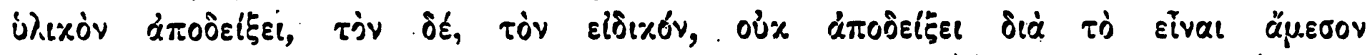
(S c hol. B.r. 244 b, 46-48; cf. P a r a p h r. R i c c a r d. ap. Wz I, 58, lin. 11 sqq.). Voir aussi $93 a, 36-b, 14$. - Sur la distinction, dans l'essence, d'une partie matérielle et d'une partie formelle, cf. $\mathrm{Ph}$ y s. II, 9 fin, $200 \mathrm{~b}, 7 \mathrm{sq}$; $\mathrm{M}$ e $\mathrm{t}$ a. $\mathrm{H}, 3,1043 \mathrm{~b}, 28-32 ; 6,1045 \mathrm{a}, 33-35 ; \mathrm{Z}, 11,1036 \mathrm{~b}, 3-7 ; 10,1036 \mathrm{a}, 9-12$; cf. $11,1037 \mathrm{a}, 4 \mathrm{sq}$.

$\left.{ }^{40}\right)$ Voir supra $\S 25$, s. in. (à propos de A n. p o s t. II, 8,93 a, 18 sq.). Cf. en outre:E t h. N i c. I, 7, 1098 b, 2. Plus haut, 1041. a, 15-18, Aristote a déjà montré que, si la recherche de la cause consistait à se demander pourquoi la chose est la chose même, la connaissance du fait en tiendrait. lieu. Voir aussi A n. p os t. I 1 et 2 . 
recherche porte sur la matière: pourquoi telle matière est-elle telle chose? Par exemple, pourquoi tels matériaux sont-ils une maison? Parce que la quiddité de la maison leur appartient comme attribut ( $\delta$ ז七

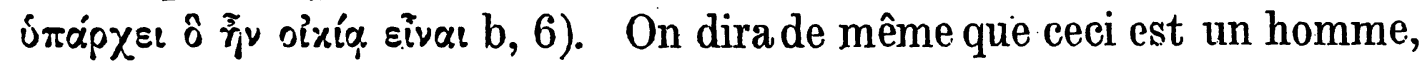
ou que ce corps, possédant telle détermination, est un homme. Par conséquent, la cause qu'on cherche, c'est la cause de la matière [ou, plus précisément, qui fait de la matiére la chose qu'elle est]; or cette chose, c'est la forme par laquelle elle est une chose déterminée, et c'est cela qui est la substance ${ }^{41}$ ). Par conséquent il est clair que, lorsqu'il s'agit des natures simples, il ne peut y avoir ni recherche, ni enseignement de ce genre, mais qu'il faut employer, à l'égard de telles natures, un autre procédé de recherche $\left.{ }^{42}\right)^{46}$. Par conséquent aussi, poursuit Aristote, la substance dont nous venons de parler est la forme, non d'un agrégat d'éléments dépourvu de toute unité, mais d'un composé dont les éléments forment un tout un. La preuve en est que, si les éléments viennent à se séparer, ce qui cesse d'exister, c'est le composé; le composé n'est done pas seulement ses éléments, mais autre chose encore. Dira-t-on que cette chose est elle-même un composé d'éléments? Le même raisonnement se représenterait alors, et ainsi à l'infini. Dira-t-on que c'est un élément? Mais il s'agit d'un composé. Il faudra done reconnaître que ce quelque chose est bien quelque chose, et qu'il est „la cause qui fait que telle chose [c'est à dire telle matière] est chair, telle autre, syllabe. De même pour les autres composés. Or cela, c'est

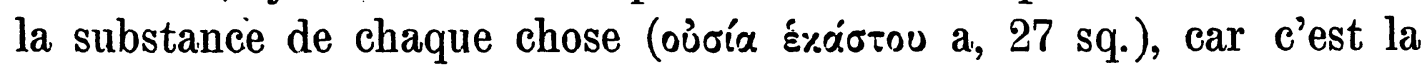
cause immédiate en vertu de laquelle elle est ce qu'elle est (aítıov

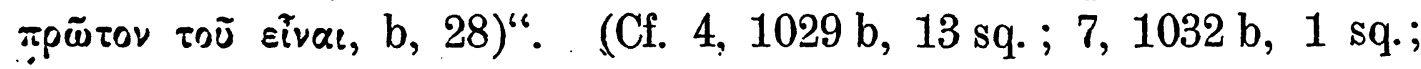
i1, 1037 a, 29 sq.)

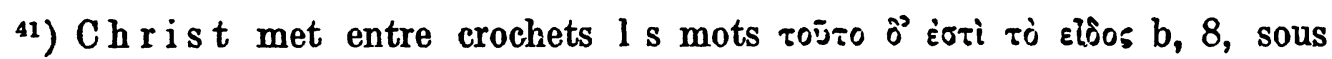

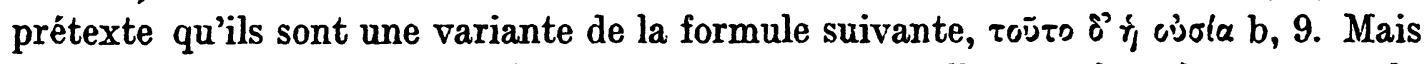
c'est à tort, semble-t-il. Aristote veut montrer en effet que la substance est la cause qui fait qu'une chose est ce qu'elle est; il fait voir d'abord que la forme est cette cause; puis il affirme que la forme c'est la substance.

42) Aristote veut dire que, dans le cas d'essences indivisibles, il ne saurait $y$ avoir de recherche de la cause. En effet, on n'y peut trouver la distinction requise d'un sujet et d'un attribut, ni. chercher des intermédiaires qui les relient l'un à l'autre. Elles ne sont pas connles par un acte de la pensée discursive, mais par une intuition indivisible ( $M$ e ta. $\theta, 10$, principalement $1051 \mathrm{~b}, 17-1052 \mathrm{a}, 4$; D e A n. III, 6 début, $430 \mathrm{a}, 26-28$ et b, 26 sqq., fin du ch. 
$\S 28$. Ainsi donc, dans tout ce qui n'est pas indivisible, il y a lieu de distinguer un élément formel et un élément matériel, quelque chose qui n'est que substratum passif et quelque chose qui agit sur ce substratum et le détermine. Mais, s'il en est ainsi, il peut arriver aussi qu'on soit préoccupé par un de ces aspects de la chose plus que que par l'autre. Si on fait attention exclusivement à la forme de la chose, on en donne une définition purement logique et vide, à laquelle fait défaut tout contenu matériel auquel la forme puisse s'appliquer: c'est ce que fait le dialecticien, quand il définit, par exemple, la colère: le désir d'offenser à son tour, ou la maison: un abri contre les intempéries. Si d'autre part on ne prend garde qu'à la matière, on définit encore d'une façon incomplète; mais ce qui manque alors, c'est la quiddité, la réalité propre de ce qu'on exprime: c'est ainsi que procède le mauvais physicien quand il dit que la colère est une vaporisation du sang qui entoure le cœur, ou que la maison, ce sont des briques, des pierres, du bois. Or ces matériaux pourraient être tout autre chose qu'une maison, recevoir une autre forme ou détermination. Il faut donc éviter un double écueil, soit d'oublier la détermination ou la forme et de n'indiquer que ce à quoi elle s'applique, soit d'indiquer. seulement la détermination ou la forme, sans dire en quoi elle se réalise. Une bonne définition est celle qui unit la forme et la matière; c'est celle du vrai physicien qui définira la maison en disant qu'elle est un abri, fait de pierres, de briques et de bois, et destiné (la fin n'est d'ailleurs pas autre chose que la forme) à nous protéger contre les intempéries. Or une'telle définition, en même temps qu'elle nous donne l'essence totale du défini, est une définition causale. C'est la seule définition vraiment scientifique, parce que, tout en tenant compte de la matière, elle met la forme au premier plan, et aussi parce que, en disant ce quie la chose est en soi essentiellement, elle nous découvre du même coup la source des attributs que cette chose possède par soi ${ }^{43}$ ).

43) D e A n. I, 1, 403 a, 25-b, 9 (cf. 402 a. 8, 15, 16-21, 25-403 a, 2); II, 2,413 a, 13-20. Dans ce dernier passage, Aristote montre qu'une définition d'où la cause est absentẹ n'est que l'énoncé d'une conclusion, à laquelle toute justification fait défaut. Ainsi, par exemple, à cette question: qu'est-ce que la quadrature . du triangle rectangle? on répond ordinairement que c'est l'équivalence de ce triangle rectangle à un carré; on définit alors par l'énoncé seul de la conclusion et on néglige d'en indiquer l a c a u s e, qui consiste dans la découverte d'une m o y e n n e proportionnelle (voir aussi $\mathrm{Me}$ e $\mathrm{a}$. B, $2,996 \mathrm{~b}, 20 \mathrm{sq}$.). On trouve des idées analogues 
§29. Cette théorie des rapports de la définition avec la connaissance de la cause se présente sous une forme trềs intéressante dans un passage des S e c onds Analytiques II, 10, $93 \mathrm{~b}$, 29-94 a, 14 (cf. I, 8, 75 b, 31 sq.). Aristote y distingue quatre sortes de définition: l'une consiste à donner simplement la signification d'un mot, la seconde est une énonciation non démontrée de l'essence, la

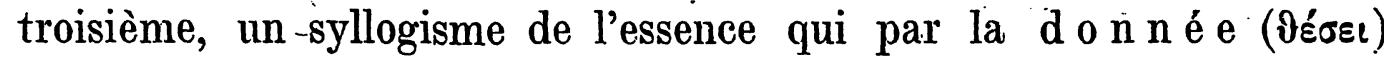
ou par le $\mathrm{mod}$ e ( $\pi \tau \tau^{0} \sigma \varepsilon \imath$ ) diffère de la démonstration, la quatrième, la conclusion de la démonstration de l'essence. Ecartons tout de suite les deux premières et la dernière. Donner la signification d'un mot, dire par exemple que le triangle, c'est une figure qui a trois angles, que l'éclipse de lune, c'est la disparition de la lune à nos regards alors qu'elle est dans son plein, que le tonnerre, e'est un bruit qu'on entend apiès l'éclair $\left.{ }^{44}\right)$, - ce n'est pas expliquer ni donner la raison de la chose exprimée par le mot. Il en est de même quand on pose l'essence sans la démontrer, ce qui est le cas pour la définition des notions immédiates et premières, et précisément parce qu'elles sont premières et immédiates. Enfin la dernière espèce de définition ne nous donne pas non plus la cause; c'est, en effet, la conclusion du syllogisme de l'essence: elle consiste à définir le tonnerre en disant que c'est un

dans Me ta. Z, 17, 1041 a, 9-b, 4: Aristote y explique que la substance est un principe et une cause et que la recherche du pourquoi consiste toujours à se demander pourquoi une certaine chose appartient comme attribut à une autre chose; cette cause c'est ou la quiddité, ou la fin, ou le moteur; ce qui fait que des briques, des pierres, du bois sont une maison, c'est ou qu'elles ont pour forme d'être un abri, ou qu'elles servent à garantir contre les intempéries, ou qu'elles ont été disposées par un architecte. Pour la suite du morceau, voir la dernière partie du $\$ 27$. - Entre la question

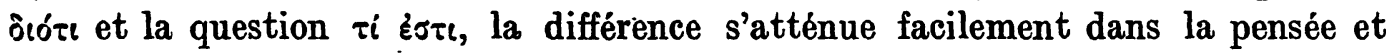
dans le langage d'Aristote; les deux questions succèdent avec la même portée et presque avec le même sens à la question ỡı. A n. p o s t. II, 2, 90 a, 14-23 (cf. Wz II, 380 sq.): „C'est le même de savoir c e qu' e s t la chose et pourquoi elle est. $Q \mathbf{u}$ ' e s t - c e qu'une éclipse de lune? C'est une privation, par l'interposition de la terre, de la lumière qui nous vient de la lune. Par q u o i l'éclipse est-elle produite, ou p o u r qu oi la lune est-elle éclipsée? $P$ a r c e qu e la lumière fait défaut, la terre s'étant interposée. $Q u$ 'est - ce que l'accord? Un rapport de nombres dáns l'aigu et le grave. En vertu de quoi l'aigu s'accorde-t-il avec le grave? Parce qu e l'aigu et le grave ont entre eux un rapport numérique." Cf. $8,93 \mathrm{a}, 3-5 ; 10,93 \mathrm{~b}, 32$.

44) Le premier exemple seul est d'Aristote, le second est de Th e m is t. I, 81,20 sq. Sp.; j'ai imaginé le troisième pour avoir un terme de comparaison avec l'exemple employé ensuite par Aristote lui-même. 
bruit dans les nuages. Or les nuages sont ici le sujet auquel on reconnaît qu'appartient le bruit du tonnerre. Cette conclusion porte donc sur la matic̀re ct elle est séparée du moyen-terme qui a servi à l'obtenir, lequel au contraire,est cause et forme (cf. n. 43 début). Puisque la cause et la forme lui font défaut, c'est bien une définition toute matérielle, et, par conséquent, entièrement opposée à la précédente

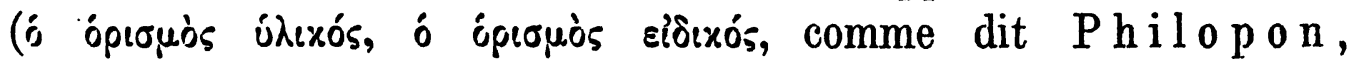
Schol. Br. 245 b, 42, 48). - Reste donc la troisième espèce, elle, nous intéresse tout particulièrement, car c'est, dit Aristote, גófos

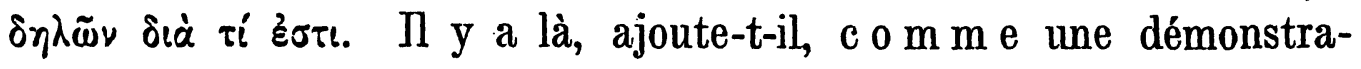
tion de l'essence. La même idée peut s'exprimer èn effet de deux façons différentes: ou bien je dirai que le tonnerre est le bruit du feu qui s'éteint dans les nuages, c'est là une définition; ou bien je dirai qu'il tonne parce que le feu s'éteint dans les nuages: ce sera la même idée présentée sous forme de dé mon stration c on t in u e ${ }^{45}$ ). La définition en question est donc l'équivalent du syllogisme logique: c'est pourquoi Aristote n'hésite pas à l'appeler, bien qu'il l'ait distin-

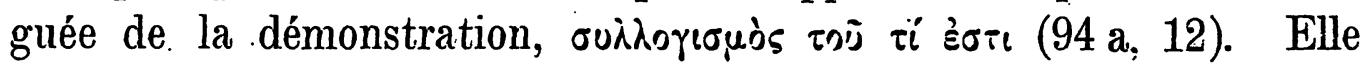
diffère, avons-nous vu, de la démonstration - ७śss! et $\pi \tau \omega \dot{\sigma \varepsilon \varepsilon . ~ C o n-~}$

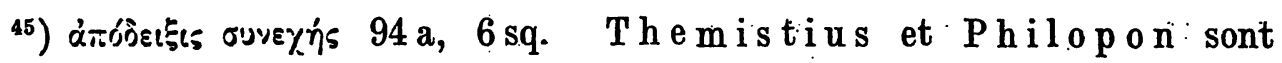
d'accord pour rapporte cette expression au fait que la démonstration, dont la définitiọn est.ici l'équivalent, se construit selon la première figure. Le premier, au lieu de présenter cette démonstration sous la forme condensée que lui donne Aristote, en développe les articulations: l'extinction du feu est un bruit de tonnerre; c'est dans les nuages que se produit l'extinction du feu; donc. c'est dans les nuages que

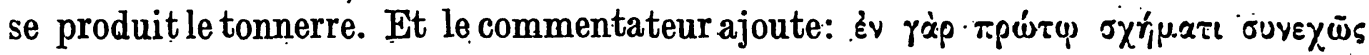

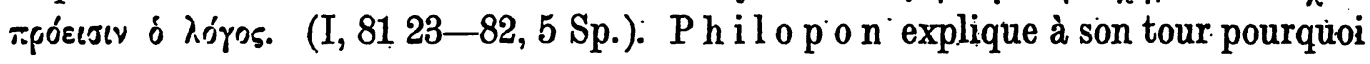

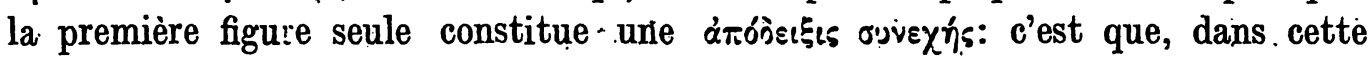
figure, les'termes forment en quelque sorte une ligne droite; au contraire, dans la seconde et dans la troisième, le moỳen est" en dehors des extrêmes .(S c h o l. $245 \mathrm{~b}, 29-32)$. Toutefois, un scoliaste anonyme, ap̀rès àvoir donné la même explication que $\mathrm{Philopoin;} \mathrm{en} \mathrm{propose} \mathrm{une} \mathrm{autre} \mathrm{qui} \mathrm{est} \mathrm{assez} \mathrm{séduisante.} \mathrm{Par}$ le mot бuve $\chi \eta_{5}$ Aristote voudrait dire que, la division des propositions et la répétition du moyen disparaissant dans une démonstration ainsi présentée, les termes y sont exposés d'une façon continue: ,parce que le feu s'éteint dans les nuages, un bruit se produit en eux qui est le tonnerre". (S c h o l. $245 \mathrm{~b}, 34-41$.) Il est certain que cette interprétation s'accorde avec la signification ordinaire du mot ouve $\chi \gamma_{i}^{\prime} s$, puisqu'il désigne la fusion en une seule des différentés partics d'une même chose.

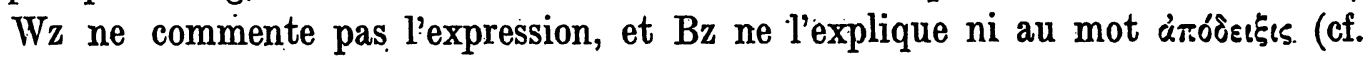

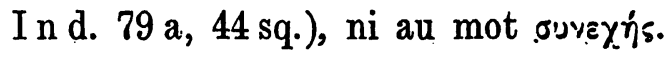


sidérons tout d'abord la première sorte de différence $\left.{ }^{46}\right)$. Lạ Ośoเs de la définition causale, sa donnée, c'est en effet ce que prouve la démonstration par une suite c on tin u e de propositions liées par la communauté du moyen-cause ${ }^{47}$ ). D'autre part cette démonstration a, elle aussi, sa ७ une démonstration véritable (cf. $n .37$ fin, $\$ 25 \mathrm{~s}$. in. et $\S 27$, second alinéa); mais elle consiste à déduire la partie matérielle de la définition de sa partie formelle. Or celle-ci est p o s é e, mais non démontrée. Mais, une fois qu'on a trouvé le moyen-terme, on est en possession d'une cause formelle et première, immédiate en elle-même, tout en étant

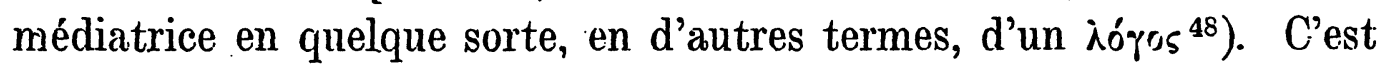

46) Géoıs paraît être pris ici $(94 \mathrm{a}, 2)$ dans le même sens qu'il a un peu plus bas $(a, 9)$, et que détermine le texte suivant de A n. p os t. I, 2, 72 a, 14-16:

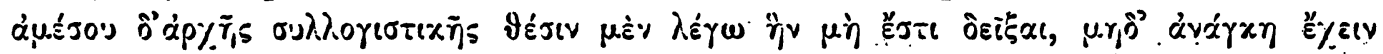

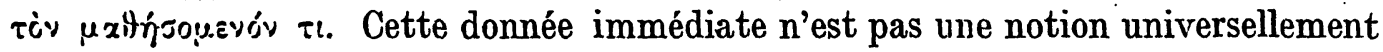
admise comme l'axiome, mais une notion que l'élève ne possède pas en son esprit,

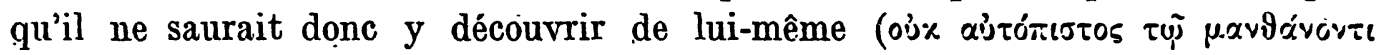
Phil o p., Schol. Br. 200 a, 13) et que le maittre doit luị fournir comme point de départ de la recherche. (Cf. Th e m. I, 10, 9-26 Sp.; Phil.o p. loc. cit. a, 11-18; T r e n d., E 1.10 g. $§ 53$ [ed. VIII, p. 140]: „ut syllogismus procedat, debet ab aliquo conveniri, quod non demonstretur.") C'est dans un sens un peu différent, mais au fond très voisin (cf. Wz II, 308), non plus le point de départ d'une

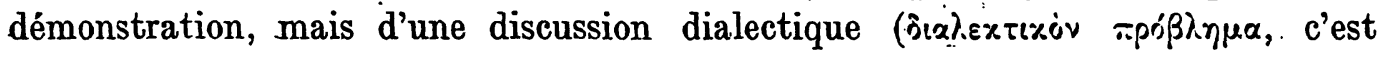
à dire la thèse sur laquelle on dispute; cf. T o p. I, 11, $104 \mathrm{~b}, 19-28,35 ; \mathrm{E} t \mathrm{~h}$. $\mathrm{N}$ i c. I, 3, $1096 \mathrm{a}, 2)$. En résumé $\vartheta \dot{\sigma \iota s, ~ c ' e s t ~, v e l ~ i d ~ q u o d ~ n o n ~ d e m o n s t r a t u m ~}$ ponitur fundamentum demonstrationis, ... vel omnino id quod statuitur ac contenditur"s. (Bz I n d. 327 b, 20 sqq., 29; Z e 11 e r II $2^{3}, 236,4$ ). Ainsi 9ćsเ5, c'est toujours le point de départ donné et non démontré.

47) Wz me parait s'exprimer inexactement quand il écrit (II, 398): „Definitio rei, qua eius causa explicatur, quasi demonstrat quid res sit et non nisi Эéozı a demonstratione differt... Qui definit, is conclusionem, quam cogit qui definitionem demonstrat, non demonstratam pronuntiat." Mais peut-on dire de la définition dont nous parlons qu'elle est constituée par la conclusion de la démonstration de l'essence? Cette formule convient seulement à la dernière espèce de définition. Plus correcte est la façon dont $W z$, à propos de $I, 8,75 \mathrm{~b}, 32$, détermine la nature de la définition causale: ,propositio quae a d monstratione non nisi eo differt, quod ipsa ponit quae haec syllogismo facto probat:" (II, 323).

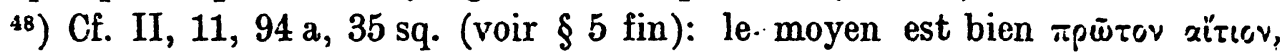

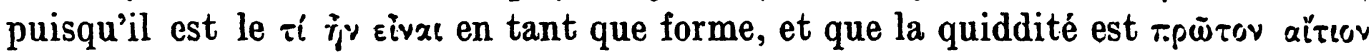

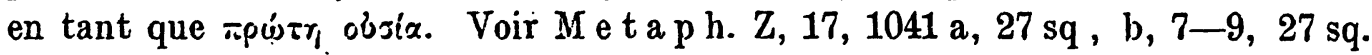
et $\mathrm{Bz}$ In d. $764 \mathrm{~b}, 21 \mathrm{sqq}$. Consulter en outre $\mathrm{Z}$ e 11 er II. $2^{3}, 161,3,4 ; 162,1$,

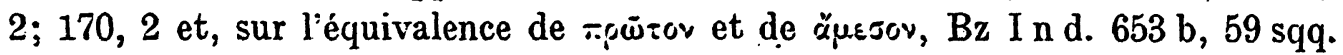


donc l'essence formelle du défini, c'est à dire la cause, qui est la Өśsıs indémontrable du syllogisme logique de l'essence. Bien différente est, comme nous l'avons vu, la Oéoıs de la définition causale; elle prend pour donnée l' e f f e t produit et le rattache à sa cause. Fille réunit alors l'essence formelle et l'essence matérielle, l'extinction du feu, qui est cause et forme, au bruit dans les nuages, qui est effet et matière, et, à ce tître, elle est supérieure au syllogisme logique de l'essence, en ce qu'elle unit ce que celui-ci séparait artificiellement ${ }^{49}$ ). Quant à la distinction qui, entre la définition causale et la démonstration del'essence, provient de la $\pi \tau \omega ́ \sigma เ \varsigma^{50}$ ), elle est analogue. La démonstration résulte d'une opération mentale dont les articulations sont

49) Cf. n. 37. Ce caractère synthétique de la définition causale a été bien

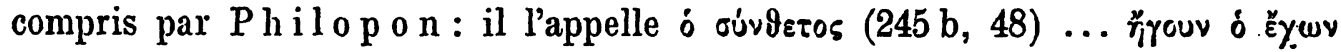

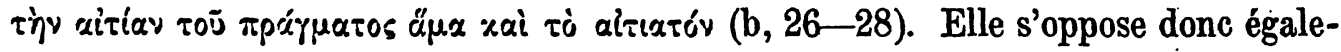
ment, comme nous l'avons déjà indiqué (cf. $§ 29 \mathrm{~s}$. in.), à la définition purement matérielle èt à la définition purement formelle, celle-ci point de départ du syllogisme logique, celle-là conclusion de ce syllogisme (cf. supra § 28). L'auteur des scolies

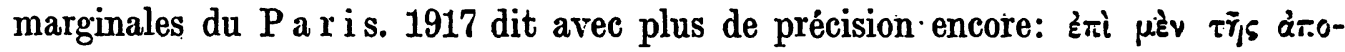

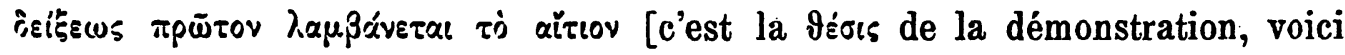

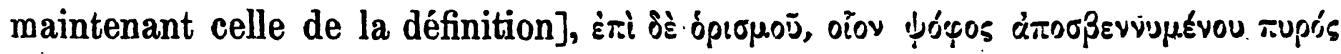

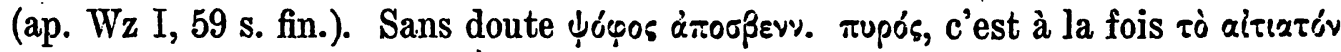

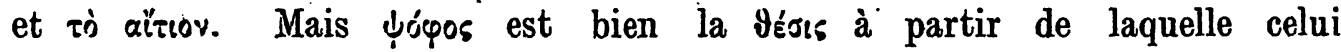

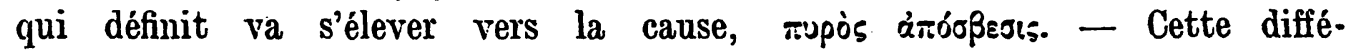

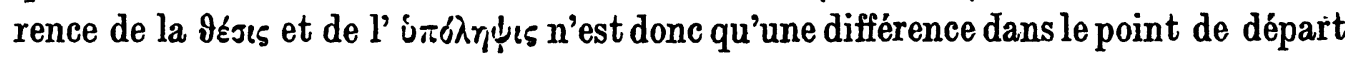
de l'opération mentale, et il ne semble pas qu'on puisse y voir, avec $\mathrm{Rod}$ i e $\mathrm{r}$ (Traité d e l'A me II, 193), une différence de la position des termes ou de leur ordre de succession dans l'énonciation verbale. En effet, dans le syllogisme logique, le moyen-cause occupe la seconde place, puisque ce syllogisme se fait dans la première figure. Or dans cette figure le moyen est sujet dans la majeure et, d'après le mode d'énonciation adopté par Aristote, c'est le sujet qui occupe la seconde place (cf. Wz II, 381, 387); il reste vrai d'ailleurs que le moyen est

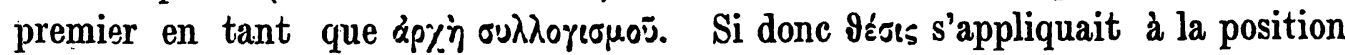
même des termes, il n'y aurait plus de différence à ce point de vue entre la démon-: stration et la définition causale, car, dans les deux cas, le premier terme apparu dansl'énonciation verbale, ce serait le majeur-effet „,bruit“; ou .,bruit de tonnerre“.

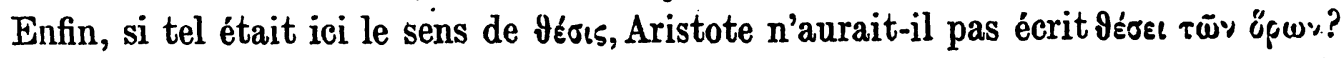

50) $\pi \approx \dot{\omega} \varsigma_{5}$, c'est tout accident de l'expression verbale correspondant aux modifications de la pensée. Ce mot désigne à la fois les diverses flexions des substantifs, des adjectifs, des verbes et aussi (A n. p r. I, 26, 42 b, 30; cf. Wz I, 329 s. med.; $\mathrm{Bz}$ In d. $659 \mathrm{a}, 22 \mathrm{sqq}$.) ce que les interprètes ont appelé трб́r.ol, c'est à dire.les modes du syllogisme. J'expression mode d'exposition traduira assez exactement le sens présent de ritísts. 
rendues distinctes par le discours et forment d'ailleurs une série continue (бove $\chi^{r_{j}}$ ). La définition causale forme au contraire une énonciation unique, et cependant elle condense dans cette unité apparente une complexité identique à celle que l'autre nous rend manifeste. Elles diffèrent donc l'une de l'autre par la forme verbale, par le mode de l'énonciation, $\pi \tau \omega ́ \sigma s i{ }^{51}$ ), ainsi que par la modalité de l'opération mentale correspondante.

$\S 30$. Ainsi le syllogisme logique a ceci de commun avec la définition la plus parfaite qu'il indique la cause du fait ou, en d'autres termes, son essence. C'est même à ce titre qu'il est appelé logique: l'essence ou la quiddité, c'est en effet pour Aristote une cause logique,

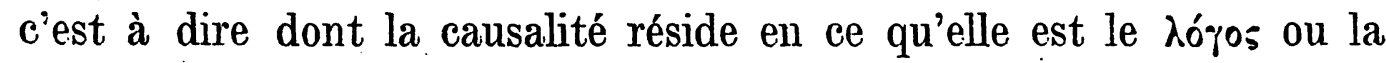
notion de la chose. En outre, tandis que, dans une vraie démonstration, on prétend non pas démontrer la substance, mais prouver qu'un attribut appartient réellement à un sujet, dans le syllogisme logique on s'efforce de donner une démonstration de la substance. Mais une telle démonstration est impossible, car elle devrait partir de cela même qu'il s'agit de démontrer et ne serait qu'une pétition de principe. On devra donc se contenter d'en donner $u$ ne sorte de démonstration, qui n'est même, à dire vrai, qu'un moyen de la rendre manifeste. Or,pour cela, nous n'avons qu'un moyen, sauf quand il s'agit d'essences indivisibles: c'est de séparer la forme ou la quiddité et, en la prenant comme moyen-terme, de conclure relativement à la matière et au sujet ${ }^{52}$ ).

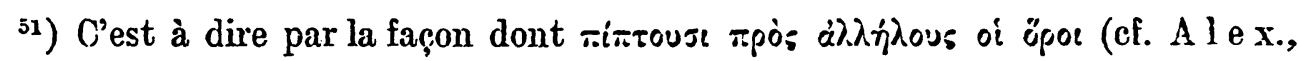
A n. p r. 287, 33 sqq. Wallies; T o p. 103, 30-104, 6, 197. 17-31 Wallies), ou

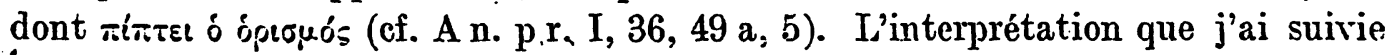
est inspirée par la remarque du scoliaste anonyme que j'ai rapportée plus haut

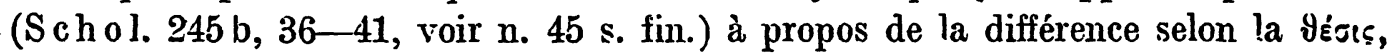
et dont $\mathrm{j}$ 'ai contesté l'application probablement vicieuse.

$\left.{ }^{52}\right)$ Voir tout ce qui précède, à partir du $\$ 25$. Sur la question de l'impossibilité de démontrer l'essence et sur la mesure dans laquelle le syllogisme logiqre peut être considéré comme fournissant une telle démonstration, voir en particulier

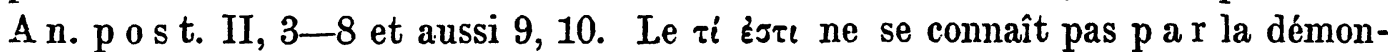
stration; mais il n'en est pas moins vrai qu'on ne peut connaître $s$ a n $s$ démon-

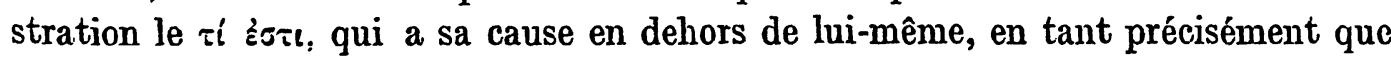
la forme est considérée alors abstraitement et à part de l'essence. A ce sujet Aristote

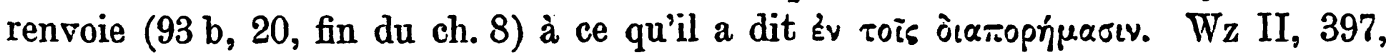
indique une référence au ch. 3. Mais cette expression, dans l'usage d'Aristote, s'applique toujours au livre $B$ de la $M$ ét a p h y s i qu e, et, en dépit des difficultés chronologiques qu'on pourrait être tenté de soulever, il faut penser qu'il en est 
Il s'ensuit que la question posée a le même sens à l'égard de la définition causale et du syllogisme de l'essence, car se demander ce qu'est une chose et se demander quelle est la raison d'être de cette chose, ce sont deux ordres de problèmes étroitement liés et qui consistent, l'un et l'autre, à se demander quel est le moyen ou à se demander quelle est la cause, ce qui revient au même; or se demander quelle est la cause, c'est se demander quelle est l'essence de la chose. Mais à cette question identique on peut, nous l'avons vu, répondre de deux manières différentes. Ou bien on prend pour donnée initiale l'effet que l'on rattache à sa cause et ainsi, dans une seule proposition, on exprime simultanément la matière et la forme de la chose; telle est, quanit au point de départ et quant au mode, la définition causale. Dans le cas du syllogisme logique, inverse est la démarche de la pensée: le principe d'où l'on part, c'est ici non plus l'effet, mais la cause et l'on énonce distinctement, d'abord la relation de la cause avec la propriété ou puissance, c'est à dire avec l'effet, puis la relation du sujet en question avec la cause, de manière à faire apparaître enfin la relation de cette propriété, c'est à dire de l'effet, avec le sujet. Par conséquent ce qui se trouvait, avec la définition causale, condensé dans l'unité d'une même proposition, se présente maintenant développé sous la forme d'une démonstration continue. L'analyse en a découvert les diverses articulations; le syllogisme les lie entre elles par une déduction régulière.

§ 31. D'autre part, dans un cas comme dans l'autre, il s'agit de rattacher une matière, qui est effet, à sa forme, qui est cause. La différence quant à l'ordre dans lequel on énonce cette relation, dans la définition causale ou dans le syllogisme logique, n'en modifie pas la nature essentielle, et la quiddité nous apparaît toujours comme étant, pour chaque chose, cause de la matière. Or ce n'est pas n'importe quelle matière qui convient ni à n'importe quelle forme, ni à telle forme particulière (cf. $\mathrm{D} \mathrm{e} \mathrm{A} \mathrm{n}$. I, 3, 407 b, 25 sq.); mais une certaine matière est déterminée par une certaine forme et c'est précisément cette détermination qui constitue la relation de cause à effet. Ce que nous venons de dire complète également nos remarques antérieures (voir §3) sur le rapport qui existe entre l'opération syllogistique et l'opération causale. Le syllogisme logique démontre l'essence à l'aide

bien de même ici; voir en effet B, 2, 997 a, 30-32. Bz dans l' In d e x omet de signaler ce renvoi $(98 \mathrm{a}, 12 \mathrm{sq}$.); cf. cependant $187 \mathrm{~b}, 4.1$. 
d'une autre essence, ou, plus exactement, l'essence matérielle (expression que la terminologie d'Aristote autorise, puisque oúría peut

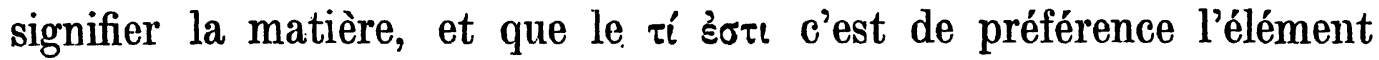
générique et matériel de la définition) à l'aide de l'essence formelle (cf. $§ 27$ s. med.). Le moyen-cause est en effet, nous le savons, l'essence propre et la quiddité du majeur et du mineur, c'est à dire de l'effet lui-même et de' son substratum (cf. $\S 5 \mathrm{~s}$. fin. et n. $10, \S 25 \mathrm{~s}$. fin. et $\S 26$. s. med.); or il possède le majeur comme attribut et est à son tour attribut du mineur; il permet donc de passer nécessairement de la forme à la matière et de rapporter avec certitude l'effet à son substratum. Enfin, on l'a vu ( $\$ 4-7)$, quel que soit le genre de causalité sur lequel porte le raisonnement, c'est toujours par l'essence formelle que l'on conclut sur l'effet relativement au sujet. Ainsi se trouve pleinement expliquée, sinon juštifiée, la célèbre formule: „Il en est [dans les générations artificielles ou naturelles] comme dans les syllogismes: e'est la substance formelle qui est le principe de tout; les syllogismes partent en effet de l'essence de la chose; or c'est aussi le cas des générations artificielles et il en est semblablement encore dans celui des générations naturelles." : (M e t a p h. Z, 9, $1034 \mathrm{a}, 30-33$; cf. $\S 2$ s. fin.) L'effet s'ensuit de sa cause comme la conséquence d'un principe. Dans toute chose, dans une éclipse de lune par exemple, il y a des propriétés fondamentales et immédiates, celles qui constituent l'essence et la font connaitre; à ces propriétés se rattachent d'autres propriétés qui sont médiates et dérivées des premières; cet ordre de dérivation, cette hiérarchie des attributs se traduit dans le syllogisme (cf. §25). Quand celui-ci est parfait et véritablement dé- monstratif, il y a convenance exacte du moyen avec le majeur et avec le mineur, et leur équivalence exprime précisément l'unité réelle de la substance, l'harmonie fondamentale de ses propriétés constitutives, matérięlles et formelles. L'effet est une puissance que la cause actualise, on l'a déjà vu ( $§ 27)$; or l'acte et la puissance ne sont pas deux choses distinctes, mais une seule et même chose, envisagée là comme puissance réalisée, ici comme tendance inachevée et possibilité de réalisation ${ }^{53}$ ).

53) Sur l'ensemble de ce développement voir A n. p o s t. II, 16, 98 b, 32-38 (fin $\mathrm{du}$ ch.). Aristote vient de montrer que, si l'effet est donné, il est nécessaire sans doute qu'il y.ait une cause, mais non que la cause existe dans sa totalité. D'où .il suit qu'une telle conclusion ne peut suffire, si on veut obtenir une démonstration vraiment scientifique (cf. $\S 11$ ). C'est pourquoi, si la question qui fait l'objet de 
\$32. A vrai dire, Aristote, on le sait, admet d'autres causes que la forme, à savoir la matière, le moteur et la fin; il va même jusqu'à déclarer que les vraies causes prochaines, c'est la matière et le moteur. Nulle part, peut-être, il ne s'explique à ce sujet avec plus de clarté que dans un passage de la Mé t a $\mathrm{p}$ h y s i q u e, $\Lambda, 5(1071 \mathrm{a}, 17-29)$. Toutes les causes, dit-il, ne peuvent pas être prises comme des universaux. Les causes prochaines de chaque chose sont, d'une part, ce qui est immédiatement en acte telle chose déterminée, c'est à dire le moteur prochain, et, d'autre part, ce qui est en puissance cette

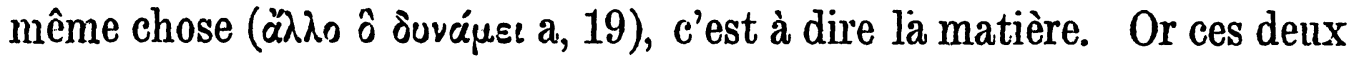
causes ne sont pas des universaux; car c'est le particulier qui est cause du particulier et chacune de ces causes est particulière, en tant que cause d'une chose particulière. En effet l'homme en général serait cause d'un homme en général. Mais il n'y a pas d'homme en général: c'est Pélée qui est cause d'Achille, votre père qui est cause de vous. $\mathrm{B}$ en général serait cause de la syllabe $\mathrm{BA}$ en général; mais c'est ce

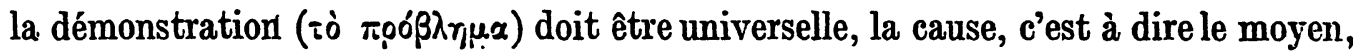
doit être prise aussi dans toute son extension puisqu'elle est le principe de la démonstration, et elle doit appartenir également à ce dont elle est la cause, c'est à dire au majeur, puisque c'est l'objet de la démonstration. Le moyen exprime en effet la quiddité du majeur (99 a, 3 sq.; cf. n. 10). Par conséquent il doit y avoir, dans tous les cas, équivalence entre le moyen et le majeur, de telle sorte que la cause et ce dont elle est la cause puissent se réciproquer. Ainsi par exemple la notion ,avoir des feuilles caduques" est déterminée universellement, quand bien même elle comporterait une diversité d'espèces, - et pour telles choses universellement, soit pour toutes les plantes, soit pour telles espèces de plantes. Pourquoi les arbres perdent-ils leurs feuilles? ' Si c'est à cause d'une coagulation de l'humide, et que tel arbre perde ses feuilles, il faut bien que a coagulation existe, et, si la coagulation existe, non pas pour n'importe quelle chose, mais pour l'arbre, la caducité des feuilles existe aussi. - Il faut bien en effet que le moyen convienno exactement aussi à ce à quoi convient le majeur et qui est le sujet de la démonstration: ilne s'agit pas, par exemple, de !'humidité du fleuve, mais de cellede l'arbre à feuilles larges (P a r a p hr. R i c c ar d.; M a r g. Par. 1917; Prodr.; cf. Wz I, 66; II, 425). Il ne doit donc y avoir dans une véritable démonstration qu'un seul moyen, qui représente la vraie cause en vertu de laquelle existe, à titre d'effet, telle propriété dạs un sujet déterminé, et ce moyen-cause, principe de la démonstration, doit pouvoir, dans une démonstration nécessaire, se réciproquer avec le majeur-effet, objet de la démonstration. Toutefois il ne faut pas oublier la réserve faite plus haut (b, 16-24, cf. § 23): toute démonstration qui, en vertu de cette réciprocité, prendra l'effet comme moyen-terme reste inférieure à l'autre qui, seule, nous fournit la raison d'être de la chose et démontre l'effet. 
B que voici qui est cause de la syllabe BA en particulier. Or ces deux causes, l'une, le moteur prochain, l'autre, la matière prochaine, sont toutes deux particulières. Maịs il est vrai qu'il y a encore d'autres causes, qui sont les formes des substances ${ }^{54}$ ). Or les causes des choses qui n'appartiennent pas au même genre, poursuit Aristote, rappelant une discussion antérieure $(1070 \mathrm{~b}, 16-21)$, sont diverses et ne sont les mêmes que par analogie. Mais, bien plus, la même diversité se rencontre entre les causes des choses qui font partie de la même espèce; sans doute ces causes ne diffèrent pas spécifiquement, mais elles sont autres cependant pour chaque chose, non seulement quaǹt à la matière et au moteur, mais aussi quant à la forme elle-même, de sorte que les causes ne sont identiques que par analogie, et seulement si, au lieu d'être envisagées par rapport à des choses particulières, elles

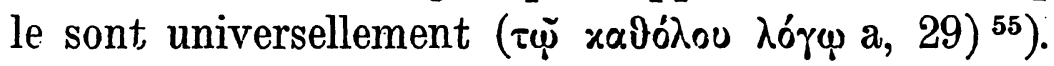

Dans le développement qui précède, Aristote s'attache surtout à montrer que telle chose particulière s'explique par une cause motrice particulière, qui en est la cause prochaine. Ailleurs (A n. p o s t. II, 18 en entier, $.99 \mathrm{~b}, 9-14$ ) il fournit une démonstration analogue en ce qui concerne la matière, et cette démonstration est d'autant plus intéressante qu'il s'agit cette fois d'expliquer, non pas une chose concrète particulière, mais bien une propriété, qui, particulière à plusieurs espèces, n'en est pas moins, à ce titre même, une sorte d'universel. C'est donc à titre d'universel que la matière va nous apparaïtre ici comme cause prochaine d'une chose particulière. Quand on envisage distinctement plusieurs espèces, explique Aristote, et qu'il y a plusieurs moyens, plusieurs causes par conséquent, on peut se demander

$\left.{ }^{54}\right)$ Et celles-ci peuvent être des universaux. J'ai adopté pour cette dernière

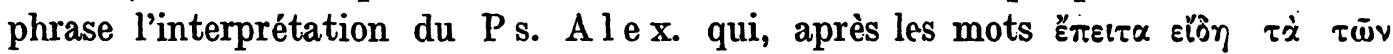

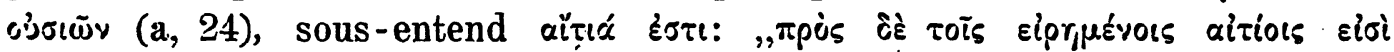

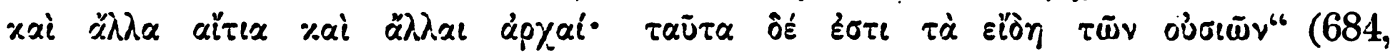
19 sq. Hayd. 657, 25-27 Bz). B on itz au contraire comprend que, pour savoir s'il faut ou non considérer les causes comme des universaux, il faut examiner si ces choses sont d'espèces diverses. Après les mots cités plus haut. il faudrait sous-entendre ópãy j̇ei, cf. a, 17. - La transposition proposée par Ch ris t ad a 18 sq. ne paraît pas suffisamment justifiée.

${ }^{55}$ ) Sur cette dernière expression, cf. Bz In d. $434 \mathrm{a}, 28 \mathrm{sqq}$. - On peut rapprocher de ce passage un autre morceau de la $M$ ét a p h. Z, 8, 1033 b, 19-34. Voir mon livre sur la théorie platonicienne des Idées et des Nombres d'après Aristote,p. 59, n. 63 . 
quelle est la véritable cause qui permet de conclure relativement a ces diverses espèces. Est-ce la cause représentée par le moyen qui est mis en relation immédiate avec l'attribut universel, c'est à dire avec le majeur? Ou bien est-ce la cause représentée par le moyen qui est, de même, en relation immédiate avec le mineur, c'est à dire avec chacune des espèces? Il est évident, répond Aristote, que la cause, c'est le moyen qui est le plus voisin de chacun des sujets dans lesquels s'exerce l'action de cette cause. C'est bien là en effet ce qui fait que le terme initial de la démonstration, celui qui se présente le premier à notre expérience, c'est à dire le mineur, est contenu dans le terme universel, c'est à dire dans le majeur. Ainsi, pour reprendre un exemple précédemment employé par Aristote ${ }^{56}$ ), la cause en vertu de laquelle la vigne et le figuier perdent leurs feuilles, c'est qu'ils sont des arbres à larges feuilles. Or expliquer ainsi, c'est expliquer par la matière. D'une part en effet la cause prochaine de la présence, dans le figuier et dans la vigne, de la propriété d'avoir des feuilles caduques, c'est qu'ils sont des arbres à larges feuilles. Mais, d'autre part, c'est là précisément le genre auquel appartiennent la vigne et le figuier, et le genre, on le sait, représente la partie matérielle de la définition de chaque chose (pour les références, voir $§ 27$, deuxième alinéa s. fin.). $\mathrm{Si}$, au contraire, on expliquait par la coagulation de la sève au point d'insertion de la feuille, on aurait une cause trop éloignée par rapport aux cas particuliers dont il s'agit; car elle convient universellement à tous les arbres qui ont la propriété de perdre leurs feuilles, propriété dont elle exprime la quiddité; mais elle n'explique nullement que la vigne et la figuier soient dans ce cas, ni pourquoi ils possèdent s $\mathrm{p}$ é $\mathrm{c}$ i a l e m e n t cette propriété. On l'expliquera au contraire en rattachant ces espèces à leur genre prochain. On pourra d'ailleurs obtenir ensuite une explication plus complète, mais aussi moins prochaine, en indiquant la cause en vertu de laquelle la propriété dont il s'agit appartient au genre.

En résumé le moteur et la matière nous fournissent des principes d'explication plus prochains que la forme. En ce qui concerne la matière, elle est cause en un double sens, cause particulière en tant qu'elle est en puissance ce que la chose particulière est en acte, cause

${ }^{\text {s6) }} 17,99 \mathrm{a}, 23 \mathrm{sqq}$. C'est ce que font $\mathrm{T}$ h e m. (I, 99, 5 sqq. Sp.) et l'auteur de la p a r a p hr. Ri c c ard., ap. Wz I, 67; cf. Wz II, 428 . Voir aussi $\$ 23$ s. in. et $\S 33$. 
aussi à titre d'universel, mais en tant que genre prochain par rapport aux espèces qui rentrent immédiatement dans ce genre. On comprend donc que la causalité de.la partie formelle de l'essence à l'égard de sa partie matérielle apparaisse à Aristote comme une causalité moins directe; en l'appelant logique, il veut dire alors qu'elle est très générale et en quelque sorte mal adaptée, en raison de cette extrême généralité, à ce qu'il s'agit précisément d'expliquer.

$\S 33$. Cependant cette façon de voir n'est pas fondamentale, et, malgré les apparences contraires, l'intellectualisme ne perd rien de son autorité sur la pensée d'Aristote. Nous l'avons vu ( $\$ 4-7$, $31 \mathrm{~s}$. med.) se servant de la distinction même des quatre espèces de causes pour prouver que la cause formelle explique toujours l'action des causes qui ne sont pas elle. Il y a plus: alors même qu'il déclare que la cause la plus prochaine de telle ou telle propriété d'une espèce, c'est le genre auquel elle appartient immédiatement, il reconnait cependant (A n. p o s t. II, 17, 99 a, 1-29) qu'il y a une cause supérieure qui explique précisément que le genre possède cette propriété: la vigne et le figuier perdent leurs feuilles, parce que ce sont des arbres à larges feuilles; mais pourquoi les arbres à feuilles larges sont-ils des arbres à feuilles caduques? C'est parce que, au point d'insertion de la feuille, il se produit en hiver une coagulation de la sève. Nous nous trouvons là en présence d'une véritable cause formelle, qui, comme nous l'avons vu dans d'autres exemples, se confond avec la quiddité même ou l'essence propre de la chose. Dans ce cas seulement ily a, sinon toujours démonstration scientifique, du moins démonstration. Comment, demande Aristote, un seul et même effet pourrait-il ne pas dépendre 'd'une seule et même cause dans tous les sujets où il se rencontre, mais ici d'une cause et là d'une antre? Si ce qui fait l'objet de la démonstration, à savoir le majeur, est démontré universellement ou comme étant un attribut nécessaire et immédiat du sujet, c'est à dire du mineur, il est impossible qu'il y ait plusieurs causes (cf. $\S 9$ ); car le moyen exprime la quiddité du majeur, et par conséquent celui-ci le possède universellement, ou, en d'autres termes, la cause (le moyen) appartient nécessairement et immédiatement à l'effet (le majeur). II est donc nécessaire qu'il n'y ait qu'une seule cause. Si, au contraire, on tentait de procéder d'une façon tout accidentelle et en prenant pour principe de la démonstration un moyen qui n'exprime pas la quiddité du majeur, alors on aurait à vrai dire mis la main non pas 
sur une cause, mais sur un simple s i g n e qui n'est pas lié nécessaire-

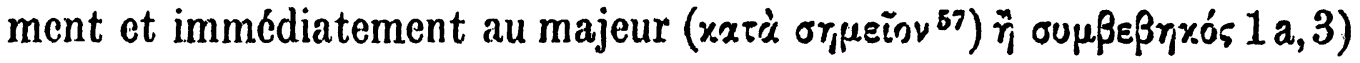
et alors il serait possible qu'une même chose dépendît, en plusieurs sujets, de plusieurs causes, et, par conséquent, qu'il y eût plus d'un moyen. Or il convient, sans doute, d'examiner le cas dans lequel le terme dont le moyen est cause (majeur-effet) et celui à l'égard duquel il est cause de la présence de l'effet à titre d'attribut (mineur-

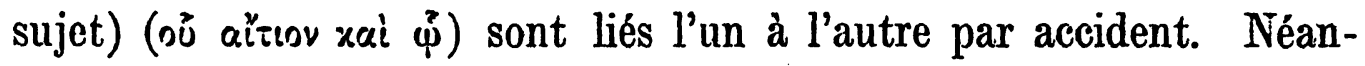
moins ce ne sont pas là des questions auxquelles on puisse répondre d'une façon vraiment démonstrative et scientifique ${ }^{58}$ ). Mais, bien qu'on ne puisse considérer comme scientifique une telle démonstration, il n'en est pas moins vrai que le rapport du moyen-terme à l'égard des extrêmes $\mathrm{y}$ traduit toujours fidèlement le rapport des extrêmes entre eux dans la conclusion (cf. $\S 12$ ). Or ce qui caractérise à cet égard la démonstration véritable, c'est l'interdépendance mutuelle des termes et la possibilité de réciproquer exactement entre eux la cause, ce dont elle est la cause et ce dans quoi elle est cause, c'est à dire le moyen, le majeur et le mineur. Si le moyen est, comme il doit être, à l'égard du majeur dans le rapport de la définition au défini, le moyen est équivalent au majeur. Considérons maintenant le mineur: il faut qu'il soit équivalent au majeur comme le moyen l'est au majeur. Or il a sans doute moins d'extension que le majeur, quand on le considère dans chacune des parties qui en constituent l'extension totale. Mais, en revanche, dès que ce même mineur est pris dans la totalité de son extension, il est équivalent au majeur. Ainsi, par exemple, ce majeur „la somme des angles extérieurs d'une figure vaut quatre droits" a plus d'extension que tel mineur particulier, soit triangle, soit carré; mais il est équivalent au mineur pris universellement, car ce mineur universel, c'est toute figure qui, étant rectiligne, a la somme de ses angles extérieurs égale à quatre droits (cf. A n. p o s t. I, 24, $85 \mathrm{~b}$,

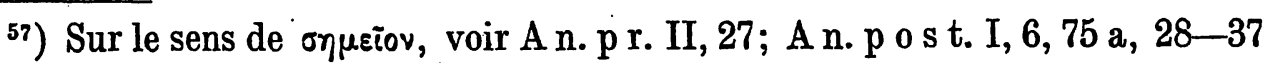
(fin du chap.); S o p h. El. 5, 167 b, 5 sqq.; R h e t. I, 2; 1357 a, 32 sqq.; Tr e n-

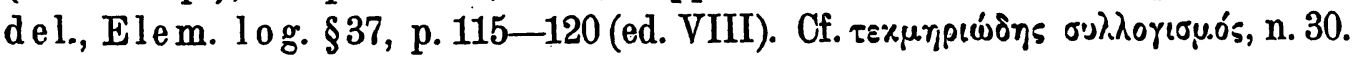

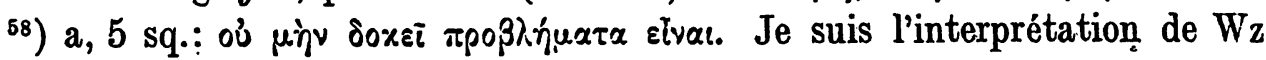

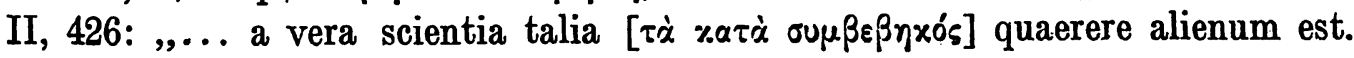

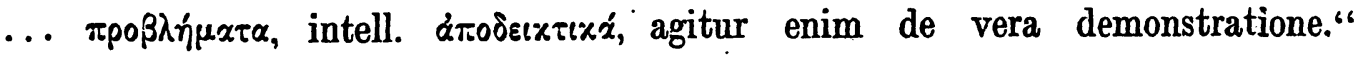
De même chez les commentateurs, $\mathrm{T}$ h e m. qui donne de bons exemples I, 97, 20 sqq. Speng., Phil o p o n (Schol. 249 b, 32 sqq.), p ar a phr. Ri c c ard. ap. Wz I, 66. - Voir aussi supra $\S 13$ et $\S 18$. 
38-86 a, 2). Or c'est précisément ce qu'exprime le majeur: le majeur est donc équivalent au mineur. Mais d'autre part le moyen donne la raison pour laquelle le majeưr, dont il exprime la quiddité, peut être affirmé, et du mineur dans sa totalité, et de chacune des parties qui sont dans l'extension du mineur. De même, dans l'exemple donné au début de ce développement, on n'a pas véritablement expliqué la caducité des feuilles dans la vigne et dans le figuiier, tant quon se contente du moyen qui nous est le plus immédiatement connu, à savoir que la vigne et le figuier sont des arbres à larges feuilles. Le mineur, la vigne et le figuier, avait alors moins d'extension que le majeur, la caducité des feuilles; mais il lui devient équivalent dès qu'on a reconnu dans la vigne et dans le figuier des arbres à larges feuilles. Quant à la raison même de cette équivalence, elle est trouvée lorsqu'on a découvert un nouveau moyen, qui est la cause primitive, à savoir la coagulation de la sève à l'endroit de l'œil de la plante; ce nouveau moyen en effet est la définition même de la quiddité du majeur, la caducité des feuilles, qui est lui-même équivalent au mineur pris dans toute son extension, le genre des arbres à larges feuilles. Nous pouvons donc conclure qu'il n'y a de démonstration véritable, ni par conséquent de science que celle dont la définition est l'instrument, et dans laquelle il y a équivalence de la cause avec son effet et avec les sujets. où l'effet se produit. Expliquer par la partie matérielle de la définition, c'est done en un sens donner une cause prochaine, mais seulement eu égard à l'insuffisance de nos recherches. La véritable explication, c'est celle qui se fait par la forme ou la quiddité, un seul et même moyen exprimant la quiddité du majeur et du mineur et fondant leur équivalence.

§34. Du reste Aristote ne se fait pas faute d'attribuer à la quiddité

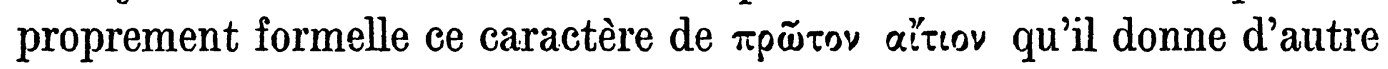
part à la matière et au moteur (voir en particulier $\S 27$ fin [M e t a. $\mathrm{Z}, 17,1041 \mathrm{~b}, 28$ ] et $n$. 48). Sans doute ce n'est pas au même sens que ces diverses causes sont premières et immédiates. Les unes sont premières dans l'ordre historique de la génération ou de la connaissance dans le temps. L'autre est première quant à la nature essentielle des choses et selon l'ordre absolu des notions. Mais qu'est-ce à dire? Elle est première de cette façon, précisément parce qu'elle n'est pas une cause particulière, parce qu'elle est un principe universel du connaitre aussi bien que de l'être. Il est à peine besoin de rappeler en 
effet que nous ne connaissons une chose que dans la mesure où nous en connaissons la quiddité ou la forme (voir par ex. M e t a. Z, 6, 1031 b, $6 \mathrm{sq}$.), et, d'autre part, si c'est Pélée qui est cause d'Achille, ce n'est pas en tant que Pélée, mais bien en tant qu'il possède la forme de l'homme: la formule classique „c'est l'homme qui engendre l'homme“ doit être entendue dans la plénitude de sa signification conceptualiste, ou même réaliste, et ce serait trahir la pensée d'Aristote que de chercher à en atténuer la portée: l'action de la nature est toute pareille à celle de l'art, la fabrication d'une chose ne s'explique que parce que la forme de la chose est déjà réalisée dans l'esprit de l'artiste; la génération d'un être, parce que la forme de cet être, ou son type spécifique, existe déjà dans son générateur. La causalité de la fabrication ou de la génération n'est au fond que l'imitation de la forme ${ }^{59}$ ).

$\S 35$. Que résulte-t-il donc de tout cela? Que la véritable cause est un universel. La forme en effet est un universel, et cela est vrai soit qu'on l'envisage en extension, ou bien en compréhension. Nous plaçons-nous au premier point de vue et considérons-nous la forme comme s'appliquant à une pluralité d'individus? Elle possède alors l'universalité en tant qu'elle est l'espèce dernière, l'essence de la chose, considérée à part de toutes les différences proprement individuelles ${ }^{60}$ ). Envisage-t-on d'autre part la forme dans sa compréhension seule et toute pluralité mise à part? Elle signifie encore l'universel, en ce qu'elle est l'essence non individuelle, mais pure et dégagée de toute matière: l'universel véritable, qui est objet de la définition, c'est la quiddité identique à la forme ${ }^{61}$ ). En vain Aristote voit-il dans la

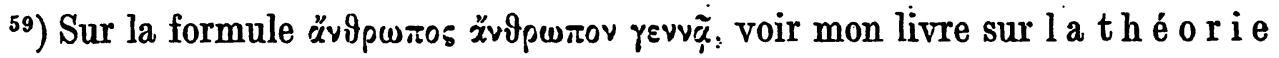
platon. des Idées et des Nombres d'après Ar., n. 63 (p. 59 sq.), p. 61 sq. et n. 67 , p. 89 et n. 97 , p. 557 et n. 513 . Sur l'interprétation de cette formule, voir principalement $110-114,116$ sq. Cf. H a m e li n, Elém. prin cipa ux de la Représentation p. 242.

${ }^{60}$ ) M e t a p h. Z, 8, 1034 a, 5-8 (fin du ch.); 12, 1038 a, 25 sq.; I, 9, 1058 b, 5-10 sqq.; B, 3, 998 b, 28 sq.; $\Delta, 101018$ b, 1-8 (fin du ch.) et al.

61) M e ta p h. Z, 10, $1035 \mathrm{~b}, 31-1036 \mathrm{a}, 2:$ il y a, dit Aristote, des parties de la forme (j'appelle forme la quiddité) et des parties du composé constitué par

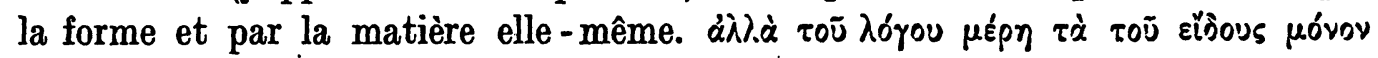

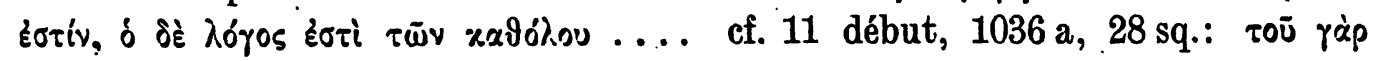

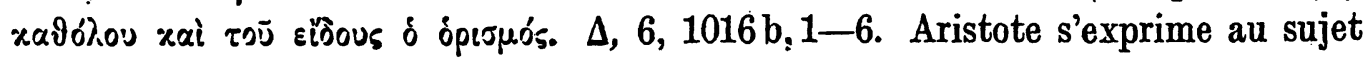

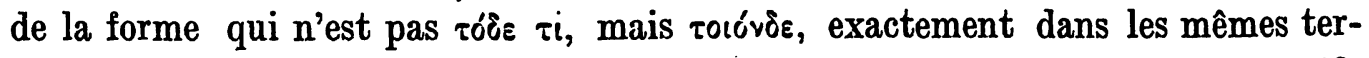
mes que au sujet de l'universel (compar. Me ta. Z, 8, 1033 b. 21-23 avec 13, $1039 \mathrm{a}, 1,15 \mathrm{sq}$.$) . Voir cependant la note suivante.$ 
forme l'essence propre de chaque chose; il n'en est pas moins vrai que, si la matière est ce qui individualise, la forme ne peut jamais être l'individualité même, mais, au plus près de l'individu, la dernière différence spécifique ${ }^{62}$ ), c'est à dire encore un universel. $\mathrm{Au}$ reste il n'y a de démonstration véritablement scientifique que celle dans laquelle le moyen convient universellement au majeur et au mineur; dans le cas même où le mineur, c'est à dire le sujet, comportant une diversité spécifique, il y aurait pareillement une diversité spécifique dans la cause, il n'y en aurait pas moins une cause universelle qui rendrait compte de ce qu'il y. a de commun dans cette diversité (cf. $\S 9$, 11, 12, 32 [deuxième partie], 33). Mais, dit Aristote, ces causes ne sont identiques que par analogie (M e ta ph. $\Lambda, 5,1071 \mathrm{a}, 17-29$, principalement 24-29, cf. § 32). Qu'importe? Les diverses causes spécifiques ne sont-elles pas elles-mêmes des causes universelles et non des causes individuelles? Elles ne diffèrent de la cause supérieure que par le degré de leur universalité. Or ce qui fait à l'égard de chaque espèce ou à l'égard de leur totalité que le moyen est véritablement cause, c'est qu'il exprime l'essence totale du majeur (cf. §33). Autrement il n'y aurait connaissance scientifique, ni de la cause, ni de l'essence de la chose sur laquelle porte la recherche. Supposons en effet que nous connaissions en même temps par la sensation et le fait à expliquer et la cause de ce fait; que, par exemple, étant placés au-dessus de la lune, nous voyions la terre s'interposer entre elle et le soleil. Or dans un cas de ce genre nous ne trouvons rien de plus qu'une simple constatation du fait, et la connaissance de la cause n'y est en aucune façon une connaissance explicative et scientifique, mais seulement le point de départ d'une telle explication. Que faudrait-

62) Voir principalement $\mathrm{Met}$ a p h. Z, 8, $1033 \mathrm{~b}, 22$ et $\mathrm{C}$ a t. $5,3 \mathrm{~b}, 18-21$ (cf. M e t a p h. Z, 11, 1037 b, 3 sq.). . C'est pourquoi, selon qu'il attribue la véritable réalité substantielle à l'individu ou bien à la quiddité (sur l'amphibologie $d u$ mot „substance“ dans la doctrine et dans la langue d'Aristote, voir mon livre déjà cité, p. 102 et n. 109), la forme est considérée par Aristote comme une substance seconde (Cat. 5, 2a, 15-19, b, 7) ou comme une substance immédiate et

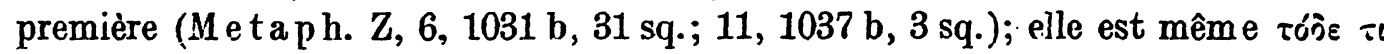
(i bid. $\theta, 7,1049$ a, 35; cf. M, 10, 1086 b, 26; D e A n. II, 1, 412a, 8; D e G e n. e t C o r r. I, 3, $318 \mathrm{~b}, 32$ ); enfin, il y a plus, au sein d'une même espèce, la forme diffère d'un individu à un autre (M eta p h. $\Lambda, 5,1071$ a, 24-29: nous ne différons pas seulement, vous et moi, par la matière, mais aussi par le moteur et par 1 a form e. Cf. $\S 32$, début.) 
il donc pour que cette constatation devînt une explication? Il faudrait que la connaissance ne résultât pas d'une simple sensation limitée à l'instant où elle se produit; il faudrait qu'elle fût universelle. La sensation ne nous fait pas connaitre le pourquoi. L'Universel, au contraire, explique les choses parce qu'il en exprime l'essence et que l'essence est la plus excellente et la plus réelle des causes ${ }^{63}$ ).

§36. Ainsi donc, pour Aristote, la cause est définitivement conçue comme identique à l'essence de la chose, en tant que forme ou quiddité, et, à ce titre, elle est un Universel. Par conséquent lä tendance analytique l'emporte en fin de compte sur la tendance synthétique, le point de vue de la logique sur celui de l'expérience. Tout ce qui est a sa cause, parce qu'il serait absurde qu'une chose fût dépourvue de la raison d'être qui réside dans son essence même (cf. $§ 23 \mathrm{~s}$. in.). $Y$ a-t-il donc une raison qui justifie, du point de vue de la philosophie générale d'Aristote, la supériorité de la tendance analytique et logique? Jusqu'à présent en effet nous n'avons fait que constater cette supériorité, nous ne l'avons pas expliquée.

La forme, remarquons le tout d'abord, est à la fois cause particulière et cause universelle. Pour expliquer chaque chose particulière, il convient en effet de faire appel à la cause la plus prochaine. Mais cette cause elle-même s'explique à son tour par une cause supérieure qui est particulière par rapport à l'autre et cependant universelle relativement à ce qu'il s'agissait en premier lieu d'expliquer. Mais cette série de formes a enfin pour terme une forme qui, n'étant unie à aucune matière (c'est l'acte pur), n'a pas besoin d'être expliquée par une forme supérieure et pour laquelle, eu égard à sa qualité d'es-

${ }^{63}$ ) Cf. A n. pos t. II, 2.90 a, 24-30 et I, 31, 87.b. 39-88 a. 2. Dans le premier de ces passages, Aristote semble admettre que, dans le cas où avec le fait nous connaitrions la cause qui le produit, cette connaissance serait suffisante, au moins en ce sens qu'il n'y aurait pas besoin de chercher un moyen-terme servant à rendre compte du fait (cf. E t h. N i c. I, 2, 1095 b, 6 sq.). Mais. dans le second texte, il déclare formellement que, dans un cas de ce genre, il n'y aurait absolument pas connaissance du òtótı, parce que l'Universel n'est pas objet de sensation. De là nous pouvons inférer, semble-t-il, que la véritable connaissance de la cause, c'est la connaissance de l'Universel, attendu que l'Universel est absolument ce qui explique les choses. Cf. O. Hamelin, Sur l'induction dans Année phil o s. X (1899), p. 41 sq:; R od i e r, Tr. d e l' A m e II, 495. Voir aussi $§ 8$. 
sence indivisible, la question de la cause ne se pose même pas (cf. n. 42). - Mais, dira-t-on, Aristote affirme expressément que la cause prochaine, c'est ou le moteur, ou la matière (cf. § 32). - Né nous occupons pas du moteur. On sait que, bien qu'il soit composé de matière et de forme, son efficacité lui vient tout entière de la forme (cf. $\S 34$ fin et n. 59). Quànt à la matière, elle ne fournit qu'une explication apparente, soit qu'on l'envisage comme étant en puissance ce que la chose doit être en acte, soit qu'on la considère comme constituée par les caractères génériques. Dans le premier cas, elle n'est que pure indétermination, tant qu'on la prend en elle-même et tout à fait indépendamment de tout rapport avec la forme qu'elle doit recevoir ${ }^{64}$ ): Au second sens il en est encore de même. Sans doute le genre ,arbres à feuilles larges" peut bien être appelé matière par rapport au figuier et à la vigne; mais c'est seulement si on envisage par abstraction la puissance, indépendamment de sa réalisation dans une forme déterminée. En réalité les caractères génériques sont inséparables de l'unité de la définition; ils font partie intégrante de la notion de l'espèce. D'ailleur's cette matière elle-même contient, à titre d'élément formel, la détermination: ,à larges feuilles", car c'est une forme par rapport à la notion d'arbre qui perd ses feuilles, et elle en exprime la quiddité. A son tour cette forme devient matière par rapport à la forme qui la définit et la détermine: ,arbres dont la sève se coagule en hiver au point d'insertion de la feuille". Par conséquent, même lorsque, en apparence, on explique par la matière, la vraie cause, c'est toujours la forme (cf. $\S 34$ début). Or la forme, c'est, nous le savons, la notion ou la quiddité de la chose. Il n'est done pas surprenant que le problème de la recherche de la cause soit essentiellement pour Aristote un problème logique et que toutes les questions particulières qu'il comporte soient traitées par lui comme s'il s'agissait proprement de relations logiques entre des concepts ${ }^{65}$ ).

64) Voir mon livre déjà cité, p. 184.

$\left.{ }^{65}\right)$ Les expressions des commentateurs grecs sont d'ailleurs très significatives.

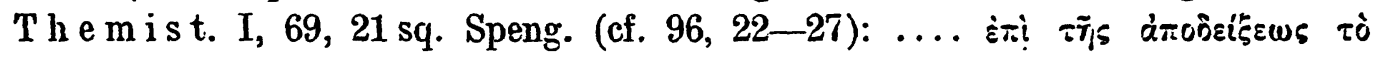

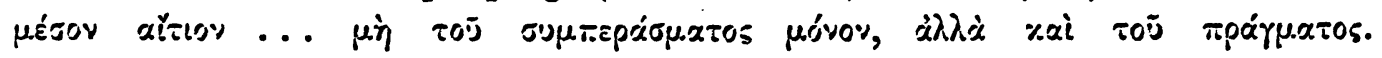

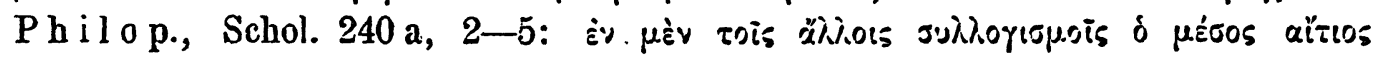

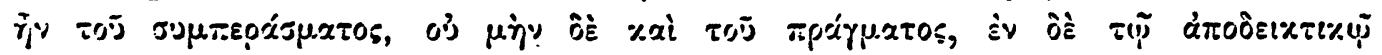

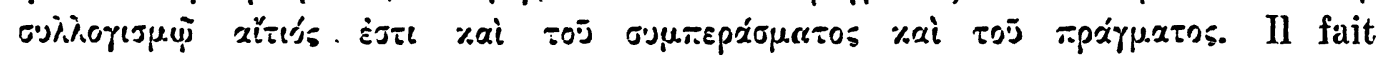
en outre remarquer, contrairement à l'opinion d' $\mathrm{Alex}$, lequel invoquait is ce sujet l'autorité de Th e o p hr., que l'objet propre du livre II des Se c onds 
D'autre part, dans l'Aristotélisme, tout le récl est constitué par un enchainement et une hiérarchie de formes. Elles se commandent en quelque sorte les unes les autres, en ce sens que chacune est plus précise en ses déterminations et, par conséquent, plus complètement intclligible que celles qui sont au-dessous d'elle. Le terme de cette hiérarchie, c'est la forme pure, qui est le déterminé même, par opposition à l'indéterminé de la puissance. C'est à la fois l'intelligible par excellence et-l'intelligence tout en acte. En elle réside donc le principe suprême de l'explication des choses; non seulement toutes les choses sont suspendues à cette chose qui seule est par elle-même; mais, bien plus, comme aucune d'elles n'est que par sa forme et que toutes les formes sont en acte dans cet intellect essentiellement actif, il s'ensuit que tout ce qui fait la réalité des choses, tout ce qui en est la raison d'être et la cause se trouve véritablement contenu dans cette cause dernière $\left.{ }^{66}\right)$. L'ordre logique des concepts est pour la pensée

An a l. n'est pas exclusivement d'exposer une théorie de la définition, mais de

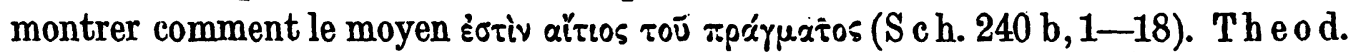
Pr o d r. (ap: Wz I, 53, 1. 9 sqq.) parle de même et observe avec beaucoup de vérité

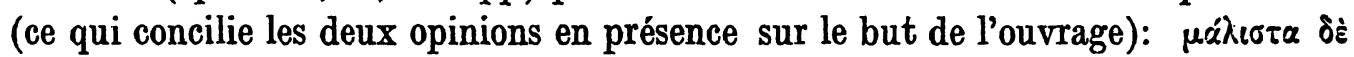

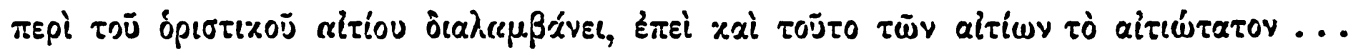
(cf. Sch. marg. Par. 1917, Schol. Br. 240 a, 47 sqq.). En effet, lisons-nous ensuite, la cause matérielle, qui est par elle-même l'indéterminé et le non-organisé, est déterminée et organisée par la cause formelle; la cause efficiente n'est pas, comme la forme ou la définition, immanente à la chose produite; enfin, tandis que la cause formelle est coexistante et innée à la chose, la cause finale se produit seulement après elle. Au reste, remonter ainsi des effets aux causes, c'est à dire du composé au simple, c'est l'objet propre de l'Analytique (cf. A l e x., . A n. p r. 7, 11 sqq. Wallies; Phil o p., Sch. 240 a, 28 sqq.; Wz 0 rg. I, 366 sq.; Trend., El. lo g., Adnotäta s. in. [p. 47 sq. ed. VIII]; Z e lle r, Ph. d. Gr. II $2^{3}$; $186,6,7)$, tandisque l'objet de l'Apodictique est de descendre des principes aux conséquences, des causes aux effets. - Voir en outre Tr e n d e l., El. lo g. §63 (ed. VIII: p. 155, 1); L o g. Unters. ${ }^{3}$, p. 31; Z eller II $2^{3}, 251$ sqq.; R o dier, De l'âm e II, 9 sq., 22-24, 490. Z e 11 e r dit excellemment p. 256: „...da die wissenschaftliche Ableitung eben in der Angabe der Ursachen zu bestehen hat, da somit jeder weitere Artunterschied eine weiter hinzutretende Ursache voraussetzt und jede solche einen Artunterschied begründet, so müßte dieses logische Gebäude der realen Abfolge und Verkettung der Ursachen genau entsprechen."

${ }^{66}$ ) Voir principalement le remarquable passage de Metaph. $\Lambda, 10$ (début), $1075 \mathrm{a}, 12-24$, et surtout $\mathrm{a}, 16-19$. Les sphères célestes et leurs moteurs, qui sont des formes séparées, forment une hiérarchie, i b i d. 8, $1073 \mathrm{a}, 26-b, 3$; De coe lo II, 10 tout entier (cf. S impl., ad loc.); 12, 292 a, 20-28; 
le symbole de l'ordre réel des essences ou des formes et celui-ci, à son tour, n'est rien autre chose que l'ordre même des causes. Si la forme suprême est appelée Dieu, nous pourrons caractériser cette construction métaphysique en disant que c'est un théisme logique. Mais si l'on songe d'autre part combien ce Dieu, bien qu'il soit un individu, est loin de posséder l'activité causale d'une réelle et vivante personnalité, on serait alors plutôt tenté de se servir d'une autre dénomination: y a-t-il aucun système auquel, par le fait même de sa doctrine de la causalité, le nom de panlogisme convienne, sinon mieux, du moins aussi bien qu'à celui-là?

§ 37. En terminant, il ne sera peut-être pas sans intérêt de comparer la conception aristotélicienne de la causalité, telle que je viens d'essayer de la définir, avec les critiques dirigées par Aristote contre la doctrine de son maître relativement au même problème. La théorie

b, 25-293 a, 2. Le terme premier, qui commande toute cette hiérarchie, est une pure forme, sans puissance ni matière, M e t a p h. $\Lambda, 8,1074 \mathrm{a}, 35$ sq.; 7, $1072 \mathrm{a}$, 24-26; b, 7 sq., 10 sq.; 13, 1073 a, 6 sq.; cf. P h y s. VIII, 10 (fin), 267 b, 17-26 (Bz In d. $390 \mathrm{~b}, 40$ sqq.). C'est un Intellect entièrement actuel et le lieu même des Intelligibles, qui sont l'objet éternellement actuel de sa pensée $(\Lambda, 7,1072 \mathrm{a}, 30 \mathrm{sq} . ; \mathrm{b}, 18-24)$. Cette forme suprême, étant dépourvue de toute puissance, est transcendante: le bien du monde, comme celui d'une armée, doit, plus encore que dans l'ordre, être dans celui qui, extérieur à cet ordre, en est le principe en sa qualité de chef. ( $\mathrm{M}$ e t a. $\Lambda, 7,1073 \mathrm{a}, 3-5 ; 8,1074 \mathrm{a}, 35 \mathrm{sq}$.; 10 début, 1075 a, 12-15; 6 tout entier; K, 2, 1060 a, 12 sq.; 7, 1064 a, 33-36; $\mathrm{Ph}$ y s. VIII passim, etc.); toutes les choses sont suspendues à ce premier principe $(\Lambda, 7,1072 \mathrm{~b}, 13 \mathrm{sq} . ; \mathrm{cf}$. $\Gamma, 2,1003 \mathrm{~b}, 16 \mathrm{sq}$.) et en expriment la nature d'une façon plus distincte ou, au contraire, plus obscure (De Co elo I, 9, 279 a, 28-30). Il est, sous tous les rapports, la cause fondamentale: en tant que cause formelle, puisqu'il contient en lui tous les Intelligibles, c'est à dire toutes les formes, et qu'il est la forme suprême; - en tant que cause motrice, puisqu'il est le premier moteur, le moteur immobile du premier ciel $(\Lambda, 7,1072 \mathrm{a}, 23,25 ; \mathrm{b}, 4,9$ sq.; 8, $1073 \mathrm{a}$, $26-30 ; 1074$ a, 36-38; P h y s. VIII, 6 (fin), 260 a, 17-19; D e C o elo II, 6, $288 \mathrm{a}, 33-\mathrm{b}, 6$; D e Ge n. et Corr. I, 3, 318 a, 1-5; II, 10, 336 b, 31-337 a, 1, 17-22); - en tant que cause finale, car il est le suprême désirable, le bien suprême vers lequel tous les êtres tendent comme vers leur fin ( $M$ e $t$ a. $-\Lambda, 7,1072$ a, 24-27, 34-b, 4; cf. a, 30-32 et D e A n. III, 10, 433 b, 15 sq.).

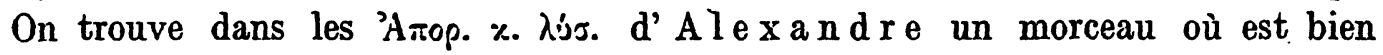
exposé le rôle de la forme suprême comme premier intelligible, en tant qu'elle est éternellement actuelle et que, de sa propre nature, elle est le simple (I, 4, 7-26. Bruns; cf. P s. A l e x. in Metaph. 687, 1-22 Hayd., 660, 14-661, 2 Bz). Voir ma théorie platoniciennedes Idées et des Nombres d'après A r is t o t.e, p. 100 sq., 105 sq., 107 sq., 155-162 et la n. 172 I, 495 sq., 583 sq. 
platonicienne de la causalité lui paraît insuffisante, parce qu'elle ne peut expliquer les choses autrement que par l'Idée: or les Idées, d'après Aristote, ne sont pas des causes suffisantes, ni de la génération ni du changement, en tant que ce sont précisément des causes formelles et non motrices, et, bien plus, elles sont plutôt des causes d'immobilité que des causes de mouvement ${ }^{67}$ ). Mais, au moins en ce qui concerne le premier point, il apparaît que la théorie aristotélicienne doit souffrir du même défaut; car elle ne nous offre pas d'autre moyen d'explication réelle et pas d'autre causalité véritable que l'essence conceptuelle ou la forme. Toutefois, tandis que cette doctrine de la causalité n'est, par rapport à l'idéalisme de Platon, le principe d'auciune incohérence, il n'en est peut-être pas de même pour la philosophie d'Aristote. Platon en effet n'hésitait pas à concilier dans l'Idée, qui est forme, l'Universèl et l'Individuel ${ }^{68}$ ). Aristote déclare, au contraire, que le principe de l'individualité ne peut être que dans la matière ${ }^{60}$ ), c'est à dire dans ces possibilités indéterminées et contingentes qui s'opposent à la détermination de la forme. Dès lors il est pour lui bien difficile, étant donné qu'il a placé dans la forme toute causalité vraie, de rendre raison de l'existence des choses individuelles: de telles choses sont, en ellesmêmes, inexplicables. Ce qui fonde leur réalité véritable, c'est en effet leur forme; mais la forme est un universel (cf. $\$ 35$, s. in.). D'autre part, ce qui fait leur individualité, c'est leur matière; mais la matière est, par elle-même, irrationnelle et inintelligible. Il n'y a donc qu'une seule individualité qui soit intelligible et qui soit rationnelle; c'est la forme suprême, l'acte pur. Ici la forme fournit l'explication de l'existence individuelle et peut en être dite la cause véritable, parce que toute matière ou puissance fait défaut. Mais, partout ailleurs, la matière, en fondant l'individualité, la rend par cela même irrationnelle, inintelligible et, comme telle, véritablement dépourṿue de cause. Que faut-il donc faire pour éviter que, dans toute chose individuelle, une partie de la chose soit, seule, véritablement expliquée, à savcir

${ }^{67}$ ) Voir mon livre déjà cité, p. 73 sqq. et principalement p. 88-94; on y trouvera réunis tous les textes relatifs à la critique d'Aristote contre cet aspect de l'idéalisme platonicien.

68) Voir particulièrement S o p h. El. 22 fin, 178 b; 36-179 a, 10; Me t a p h. M, 9, 1086 a, 32-35; cf. Z, 16, 1040 b, 27-29. Cf. mon livre, p. 34 sq. et la note 33 .

${ }^{69}$ ) Sur l'individuation par la matière, cf. Bz In d. 786 a. 52 sqq.; Z e 1 le r, P h. d. Gr. II $2^{3}, 342$ sq. 
précisément la partie non-individuelle, parce que, seule, elle peut être rattachée à la causalité de la forme? Il faut admettre que la forme pouvant être, une fois et pour un être, le principe de l'individualité peut l'être d'autres fois, et pour tous les êtres. Les formes, ou les intelligibles, contenus dans la Pensée qui se pense elle-même, deviennent par là des individus et ont dans la distinction de leurs quiddités la raison même de leur individualité. Ainsi donc, dans l'Áristotélisme, la forme se trouve être principe d'individuation, en tant précisément qu'universel, et au moins à l'égard de l'Intellect divin et de ses objets ${ }^{70}$ ). D'autre part, pourquoi la forme des formes est-elle justement l'Individu suprême? Parce qu'en elle le rapport des formes est entièrement simple et nécessaire ${ }^{71}$ ). Par suite les autres substances individuelles dépendent de la substance première et se rapprochent d'elle dans la mesure où elles réussissent mieux à réaliser le simple et le nécessaire par l'entrecroisement des formes qui les constituent. La hiérarchie décroissante des individus se mesure donc par le progrès en eux de la complexité et de la contingence. Brefla philosophie d'Aristote comporte une conception de la causalité tout à fait analogue à celle qu'il a condamnée chez son maître: la relation causale apparaît alors en effet comme une participation plus ou moins confuse à des formes universelles, dont l'existence est en fin de compte une existence séparée, puisque l'Intellect, qui est le lieu de ces formes universelles, est luimême séparé ${ }^{72}$ ). Or on ne peut nier qu'une telle doctrine ne s'accorde au mieux avec la conception logique et analytique qu'Aristote s'est faite de la causalité.

;0) La raison pour laquelle l'être en tant qu'être est à la fois individuel et universel est nettement indiquée dans Meta. E, 1 (fin du ch.), 1026 a, 23-32. Il s'agit de la question de savoir comment la philosophie première peut porter sur l'individuel, qui seul est réel, et en même temps avoir pour objet l'universel, ce qui est nécessaire pour qu'elle soit une science; mais, poursuit Aristote, s'il y a une substance immobile, cette substance est première, antérieure aux substances physiques, et il faut, à cause d'elle. qu'il y ait une philosophie première

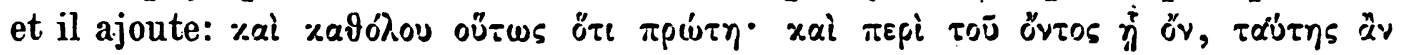

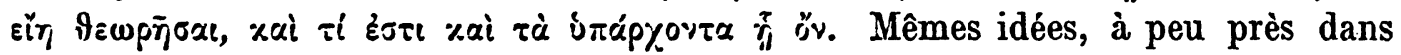
les mêmes termes, dans $\mathrm{K}, 7,1064 \mathrm{~b}, 6$-14 (cf. B, 6, $1003 \mathrm{a}, 6$-17 [fin du ch.] et $\mathrm{K}, 2,1060 \mathrm{~b}, 20-23$ ).

i1) Aristote établit une équivalence entre l'ê-soi, le simple et le nécessaire d'une part (Metaph. $\Delta, 5,1015 \mathrm{~b}, 11-13 ; \Lambda, 7,1072 \mathrm{~b}, 13 ;$ A n. p ost. I, 6, 74 b, 6 sq., 75 a, 28-31; cf. 4,73 a , 34-37), et l'universel d'autre part ( A n. p o s t. I, 4, 73 b, 26-74 a, 3 [fin du chap.]; M e t a ph. $\Delta, 9,1017 \mathrm{~b}, 35$ ).

72) Voir mon livre déjà cité, p. 110 sq., 116 sqq., 196 sq.

Archiv für Geschichte der Philosophie. XXIII. 2. 
210 Léon Robin, Sur la conception aristotélicienne de la causalité.

Mais, d'un autre côté, il semble parfois s'être avisé, nous l'avons vu, que réduire la relation causale à une identité logique, comme celle qui constitue le syllogisme, c'est peut-être en donner la traduction la plus intelligible, mais à la condition toutefois de la simplifier d'une façon factice et d'en retrancher par abstraction tout ce qu'elle a d'individuel et de concret. Ainsi donc c'est parce qu'Aristote est préoccupé de se rapprocher de l'expérience qu'il abandonne, presque involontairement et à son insu, le pỏint de vue logique pour représenter la causalité comme une relation synthétique. L'effet cesse d'être considéré comme c on te n u dans la cause; il apparait comme c on ditionné nécessairement par l'action de-celle-ci. Ces modifications passagères de l'attitude d'Aristote prouvent du moins ceci: il a senti que, dans la causalité, il y a quelque chose qui ne se démontre pas, mais qui se constate, à savoir ce qu'il y a d'individuel dans la relation causale. A ce point de vue la matière et le moteur pourraient certainement fournir à Aristote un principe d'explication. Mais il faudrait d'abord pour cela que la matière ne fût pas indétermination pure; car alors toute détermination vraie ne peut venir que de la forme, et ainsi la matière, à la vérité, n'explique rien du tout. II faudrait en second lieu que la cause efficiente, ou le moteur, ne se confondît pas avec la cause formelle ou avec la fin, identique elle-même à la forme.

En résumé il y avait chez Aristote le germe d'une interprétation positive de la causalité, qui, sans être purement formelle, restât néanmoins rationaliste. Je n'ai pas à examiner quelle serait la valeur de cette explication. Voici seulement ce que j'ai voulu montrer. D'une part, avec les tendances logiques de son idéalisme intellectualiste, Aristote ne pouvait trouver dans la matière et le moteur des principes positifs d'explication; une doctrine analogue à la participation platonicienne s'imposait donc à lui: l'effet existe parce qu'il reçoit en lui la forme de la cause ou les formes des diverses causes qui concourent à le constituer. D'autre part, en concevant la causalité comme une relation synthétique, en accordant à la matière et au moteur une puissance de détermination et une efficacité propres, Aristote a montré un sentiment très-net des exigences de la méthode expérimentale dans les sciences de faits; mais en revanche il était, par là même, en contradiction avec d'autres tendances qui, cela est indéniable, sont prédominantes dans sa philosophie. 